\title{
On obtaining Boltzmann-distributed
}

\section{configurational ensembles from Expanded Ensemble simulations with fast state mixing}

\author{
Sebastian Wingbermühle* and Lars V. Schäfer* \\ Theoretical Chemistry, Ruhr University Bochum, D-44780 Bochum, Germany \\ E-mail: Sebastian.Wingbermuehle@rub.de; Lars.Schaefer@rub.de
}

All simulations were performed using the simulation package GROMACS, version 5.0.6 $6^{1-7}$ that was extended with the implementation of the hybrid MC/MD sampler described in this work. In expanded ensemble simulations, GROMACS version 5.0.6 does not scale bonded interactions nor does it update the EXE state if the new state is state zero (i.e., the lowest state). In addition, the frequency with which the Andersen-massive thermostat is applied can vary slightly from the user's input due to rounding errors. Those three bugs were fixed in-house in version 5.0.6 before performing the simulations reported here; equivalent changes were included in the official GROMACS 2016.3 release. In addition, the input parameter nstexpanded was always chosen to be a multiple of nstcalcenergy to avoid using outdated energy values. This latter issue was fixed in GROMACS version 2018.4. Alanine dipeptide was described with the AMBER99SB* force field ${ }^{8-10}$. The TIP4P-2005 ${ }^{11}$ water model was used. 


\section{Potential energy scaling}

For the scaling ${ }^{12}$, the potential energy of the system was split into the internal potential $V_{s s}$ of the heated solute (alanine dipeptide), the interfacial solute-solvent potential $V_{s w}$, and the internal potential $V_{w w}$ of the solvent:

$$
V=V_{s s}+V_{s w}+V_{w w}
$$

$V_{s s}$ was scaled by the ratio of the inverse effective temperature of state i, $\beta_{i}$, and the system's inverse temperature $\beta$, whereas $V_{s w}$ was only scaled by the square root of this factor:

$$
\begin{aligned}
V_{i} & =\frac{\beta_{i}}{\beta} V_{s s}+\sqrt{\frac{\beta_{i}}{\beta}} V_{s w}+V_{w w} \\
& =\gamma_{i} V_{s s}+\sqrt{\gamma_{i}} V_{s w}+V_{w w}
\end{aligned}
$$

with the linear interpolation

$$
\beta_{i}=\left(1-\lambda_{i}\right) \beta+\lambda_{i} \beta_{H}
$$

where $\beta_{H}$ denotes the inverse of the highest effective temperature and $\lambda_{i}$ is the value of the coupling parameter $\lambda$ at state i. Accordingly, force constants for bonds, angles, dihedrals, and improper dihedrals belonging to the heated solute were scaled by $\gamma_{H}$. Due to the combination rule applied by the AMBER force field, $\epsilon_{j k}=\sqrt{\epsilon_{j} \epsilon_{k}}$, Lennard-Jones well depths $\epsilon_{j}$ belonging to the solute were scaled by $\gamma_{H}$, too. Partial charges belonging to the solute were scaled by $\sqrt{\gamma_{H}}$. To carry out the potential energy scaling, the free energy perturbation (FEP) code implemented in GROMACS was used. 


\section{Details of expanded ensemble (EXE) simulations}

\subsection{Setup and equilibration}

After energy minimization in vacuum (500 steps of steepest descent), alanine dipeptide was solvated in 522 TIP4P-2005 ${ }^{11}$ water molecules with $2 \mathrm{Na}^{+}$and $2 \mathrm{Cl}^{-}$ions in a periodic rhombic dodecahedron box before the energy was minimized again by 500 steps of steepest descent. The system was equilibrated in the $\mathrm{NpT}$ ensemble at effective temperatures of $300 \mathrm{~K}, 400 \mathrm{~K}$, and $600 \mathrm{~K}$, applying harmonic position restraints with force constant $k=1000 \mathrm{kJmol}^{-1} \mathrm{~nm}^{-2}$ to alanine dipeptide. Using a 4 fs time step, configurations were propagated by the leapfrog MD integrator for $1 \mathrm{~ns}$ and saved to disc every 2500 simulation steps. Hydrogen atoms were represented by virtual sites and all bonds were constrained using $\operatorname{LINCS}^{13}$ for alanine dipeptide and SETTLE ${ }^{14}$ for the water molecules. Periodic boundary conditions were applied in $\mathrm{x}-, \mathrm{y}$ - and z-direction. Lennard-Jones $(12,6)$ interactions were calculated up to a cut-off $r_{c u t}=1 \mathrm{~nm}$ using a buffered pair list ${ }^{15}$; long-range Coulomb interactions were treated by the Particle-Mesh-Ewald method ${ }^{16}$, employing the same cut-off to discern between real and reciprocal space. The temperature was maintained at $300 \mathrm{~K}$ by one velocity rescaling thermostat with a stochastic term ${ }^{17}$ with time constant $\tau_{T}=0.1 \mathrm{ps}$. The average pressure was kept at 1 bar by one isotropic Berendsen barostat ${ }^{18}$ with time constant $\tau_{p}=1.6$ ps. Initial velocities were drawn randomly from a Maxwell-Boltzmann distribution at $300 \mathrm{~K}$. The final configurations generated here were used as starting structures for all simulations described below.

\subsection{Determination of the biasing weights}

The biasing weights were pre-equilibrated in three $10 \mathrm{~ns}$ EXE runs in the NVT ensemble. Here, the velocity Verlet integrator was employed. The temperature was maintained by

one Nosé-Hoover ${ }^{19,20}$ chain including ten thermostats with time constant $\tau_{T}=0.5 \mathrm{ps}$. The position restraints on alanine dipeptide were removed. Else, the same simulation parameters 
as during equilibration were used. Moves to a new effective temperature were attempted every 10 ps and mediated by Metropolized-Gibbs sampling ${ }^{21}$ among all states. The initial biasing weights were set to zero and updated by the Wang-Landau algorithm ${ }^{22}$ with an initial incrementor $\Delta_{W L}=3.0 \mathrm{k}_{\mathrm{B}} \mathrm{T}$. Whenever the histogram of states was considered flat, i.e., no state was populated less than $80 \%$ or more than $125 \%$ of the average, the incrementor was multiplied by 0.8 . The biasing weights were considered pre-equilibrated if the incrementor dropped below 0.0001 .

\subsection{Production runs}

In the production runs (50 ns EXE-MC/MD and EXE-MD simulations), the integrator time step was reduced to $2 \mathrm{fs}$. The initial biasing weights were chosen to be $0.0 \mathrm{k}_{\mathrm{B}} \mathrm{T}, 17.0 \mathrm{k}_{\mathrm{B}} \mathrm{T}$, and $34.0 \mathrm{k}_{\mathrm{B}} \mathrm{T}$ for the effective temperatures $300 \mathrm{~K}, 400 \mathrm{~K}$, and $600 \mathrm{~K}$, respectively. To finetune the biasing weights at the beginning of the simulation, they were updated again by the Wang-Landau algorithm. The initial Wang-Landau incrementor was set to $\Delta_{W L}=$ $1.0 \mathrm{k}_{\mathrm{B}} \mathrm{T}$. The biasing weights were considered equilibrated and not changed anymore if $\Delta_{W L}<0.05 \mathrm{k}_{\mathrm{B}} \mathrm{T}$; the criterion was met after $0.1 \mathrm{~ns}$ to $2 \mathrm{~ns}$ of simulated time. Configurations sampled during this short equilibration were discarded. In the 90 hybrid MC/MD runs, no additional thermostat was used. With the simulation parameters chosen, the probability for a proposed configuration to be accepted was $P_{\text {accept }} \approx 80 \%$. In the MD runs, the temperature was maintained by either one Andersen-massive thermostat ${ }^{23}$ (90 runs) whose time constant $\tau_{T}$ was adapted to the time interval $\tau$ between two subsequent EXE state updates or one velocity-rescaling thermostat with a stochastic term with time constant $\tau_{T}=0.1 \mathrm{ps}$ or $\tau_{T}=$ 0.5 ps (90 runs each) or one Nosé-Hoover chain including ten thermostats with time constant $\tau_{T}=0.5 \mathrm{ps}$ (90 runs). The Andersen-massive thermostat randomly assigned new MaxwellBoltzmann-distributed velocities to all particles every $\tau_{T}$. The other simulation parameters were as described in section 2.2. 


\section{Details of the standard MD simulations}

The equilibration run at the effective temperature $300 \mathrm{~K}$ yielded the starting structure for the $10 \mu \mathrm{s}$ standard MD simulation. Using a 4 fs time step, configurations were propagated in the NVT ensemble by the velocity Verlet MD integrator and saved to disc every 10 ps. Constraints on bonds, periodic boundary conditions, and Lennard-Jones and Coulomb interactions were treated in the same way as during equilibration. The temperature was maintained at $300 \mathrm{~K}$ by one Nosé-Hoover chain including ten thermostats with time constant $\tau_{T}=0.5 \mathrm{ps}$. Initial velocities were drawn randomly from a Maxwell-Boltzmann distribution at $300 \mathrm{~K}$. 
Table S1: Relative populations of four regions in the Ramachandran $\phi, \psi$ map of alanine dipeptide, corresponding to the indicated four secondary structure elements. List of the data that are shown in Fig. 2: a) hybrid MC/MD sampler, b) velocity Verlet and Andersenmassive thermostat, c) velocity Verlet and velocity-rescaling thermostat with a stochastic term, d) velocity Verlet and Nosé-Hoover chain (with ten thermostats), e) $10 \mu$ standard MD simulation with velocity Verlet MD integrator and Nosé-Hoover chain (with ten thermostats). $\tau$ and Pop. denote the EXE state update interval and the relative population of the secondary structure, respectively.

a)

\begin{tabular}{lllllllll}
\hline$\tau[\mathrm{ps}]$ & $\beta$-sheet & \multicolumn{3}{c}{ PP-helix } & & $\alpha_{\mathrm{r}}$-helix & \multicolumn{3}{c}{$\alpha_{\mathrm{l}}$-helix } \\
& Pop. & Error & Pop. & Error & Pop. & Error & Pop. & Error \\
\hline 0.2 & 0.27 & 0.01 & 0.35 & 0.01 & 0.14 & 0.01 & 0.020 & 0.007 \\
0.4 & 0.27 & 0.01 & 0.35 & 0.01 & 0.147 & 0.008 & 0.022 & 0.007 \\
0.6 & 0.28 & 0.02 & 0.36 & 0.01 & 0.14 & 0.01 & 0.020 & 0.006 \\
1.0 & 0.267 & 0.007 & 0.35 & 0.01 & 0.148 & 0.009 & 0.019 & 0.006 \\
2.0 & 0.268 & 0.004 & 0.35 & 0.01 & 0.142 & 0.006 & 0.019 & 0.002 \\
5.0 & 0.272 & 0.009 & 0.34 & 0.02 & 0.146 & 0.009 & 0.022 & 0.006 \\
\hline
\end{tabular}

b)

\begin{tabular}{|c|c|c|c|c|c|c|c|c|}
\hline \multirow[t]{2}{*}{$\tau[\mathrm{ps}]$} & \multicolumn{2}{|c|}{$\beta$-sheet } & \multicolumn{2}{|c|}{ PP-helix } & \multicolumn{2}{|c|}{$\alpha_{r}$-helix } & \multicolumn{2}{|c|}{$\alpha_{1}$-helix } \\
\hline & Pop. & Error & Pop. & Error & Pop. & Error & Pop. & Error \\
\hline 0.2 & 0.273 & 0.007 & 0.36 & 0.02 & 0.136 & 0.009 & 0.023 & 0.003 \\
\hline 0.4 & 0.27 & 0.01 & 0.35 & 0.01 & 0.142 & 0.009 & 0.021 & 0.006 \\
\hline 0.6 & 0.270 & 0.008 & 0.35 & 0.01 & 0.139 & 0.006 & 0.025 & 0.004 \\
\hline 1.0 & 0.275 & 0.004 & 0.351 & 0.004 & 0.141 & 0.003 & 0.020 & 0.002 \\
\hline 2.0 & 0.270 & 0.007 & 0.347 & 0.005 & 0.143 & 0.007 & 0.023 & 0.008 \\
\hline 5.0 & 0.27 & 0.01 & 0.345 & 0.004 & 0.144 & 0.005 & 0.020 & 0.003 \\
\hline
\end{tabular}

c)

\begin{tabular}{|c|c|c|c|c|c|c|c|c|}
\hline \multirow[t]{2}{*}{$\tau[\mathrm{ps}]$} & \multicolumn{2}{|c|}{$\beta$-sheet } & \multicolumn{2}{|c|}{ PP-helix } & \multicolumn{2}{|c|}{$\alpha_{\mathrm{r}}$-helix } & \multicolumn{2}{|c|}{$\alpha_{1}$-helix } \\
\hline & Pop. & Error & Pop. & Error & Pop. & Error & Pop. & Errol \\
\hline 0.2 & 0.280 & 0.005 & 0.352 & 0.004 & 0.138 & 0.005 & 0.021 & 0.002 \\
\hline 0.4 & 0.27 & 0.01 & 0.349 & 0.008 & 0.144 & 0.009 & 0.022 & 0.002 \\
\hline 0.6 & 0.274 & 0.009 & 0.35 & 0.01 & 0.142 & 0.007 & 0.022 & 0.005 \\
\hline 1.0 & 0.277 & 0.008 & 0.349 & 0.004 & 0.140 & 0.005 & 0.021 & 0.007 \\
\hline 2.0 & 0.276 & 0.004 & 0.354 & 0.005 & 0.136 & 0.004 & 0.021 & 0.005 \\
\hline 5.0 & 0.277 & 0.008 & 0.35 & 0.01 & 0.137 & 0.006 & 0.022 & 0.003 \\
\hline
\end{tabular}

d)

\begin{tabular}{lllllllll}
\hline$\tau[\mathrm{ps}]$ & $\beta$-sheet & & PP-helix & & $\alpha_{\mathrm{r}}$-helix & & $\alpha_{\mathrm{l}}$-helix \\
& Pop. & Error & Pop. & Error & Pop. & Error & Pop. & Error \\
\hline 0.2 & 0.265 & 0.008 & 0.329 & 0.005 & 0.156 & 0.003 & 0.024 & 0.006 \\
0.4 & 0.274 & 0.004 & 0.330 & 0.009 & 0.150 & 0.005 & 0.021 & 0.003 \\
0.6 & 0.274 & 0.003 & 0.34 & 0.02 & 0.149 & 0.009 & 0.019 & 0.004 \\
1.0 & 0.264 & 0.004 & 0.343 & 0.007 & 0.152 & 0.005 & 0.020 & 0.004 \\
2.0 & 0.269 & 0.008 & 0.346 & 0.007 & 0.144 & 0.003 & 0.025 & 0.003 \\
5.0 & 0.27 & 0.01 & 0.35 & 0.01 & 0.14 & 0.01 & 0.020 & 0.006 \\
\hline
\end{tabular}


Table S1: Relative populations of four regions in the Ramachandran $\phi, \psi$ map of alanine dipeptide (continued).

\begin{tabular}{|c|c|c|c|c|c|c|c|}
\hline$\beta$-sheet & & PP-helix & & $\alpha_{\mathrm{r}}$-helix & & $\alpha_{1}$-helix & \\
\hline Pop. & Error & Pop. & Error & Pop. & Error & Pop. & Error \\
\hline 0.2725 & 0.0003 & 0.348 & 0.002 & 0.144 & 0.002 & 0.017 & 0.005 \\
\hline
\end{tabular}

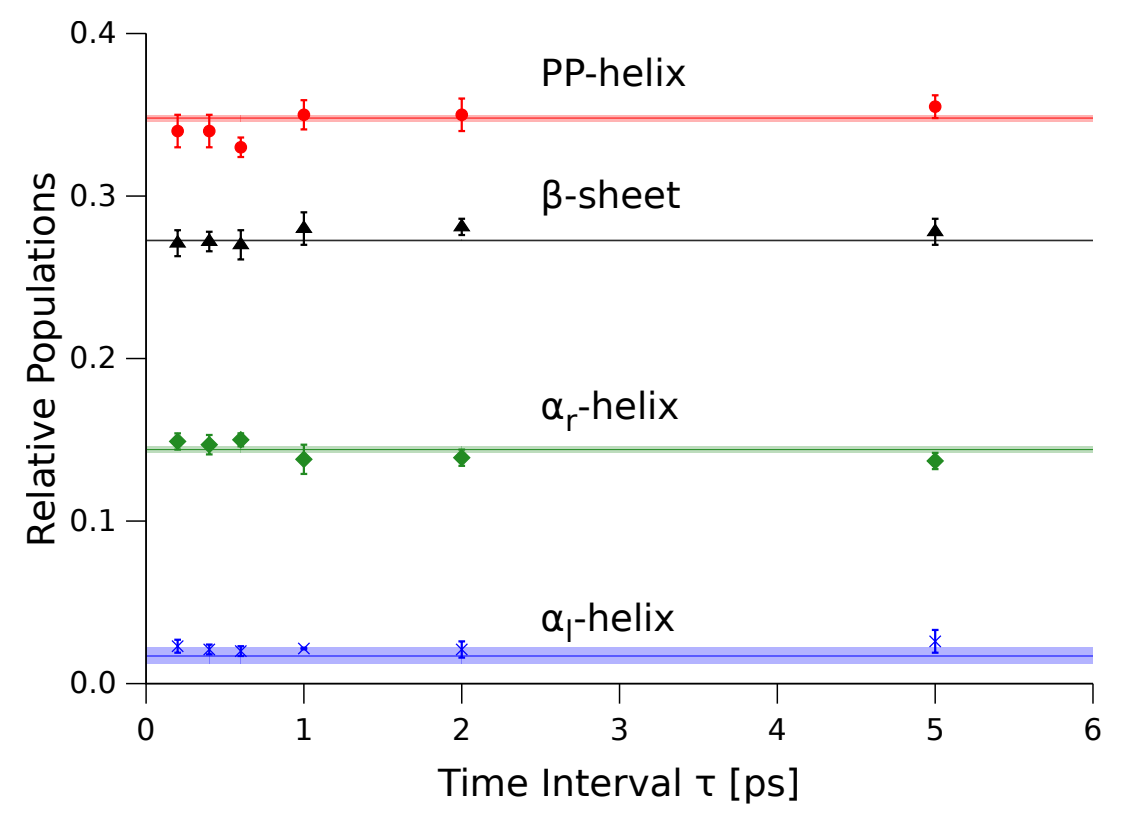

Figure S1: Relative populations of the four secondary structure elements of alanine dipeptide for the setting: integrator $=$ velocity Verlet, thermostat $=$ velocity-rescaling with a stochastic term, $\tau_{T}=0.5 \mathrm{ps}$.

Table S2: Relative populations of the four secondary structure elements of alanine dipeptide for the setting: integrator $=$ velocity Verlet, thermostat $=$ velocity-rescaling with a stochastic term, $\tau_{T}=0.5$ ps. List of the data that are shown in Fig. S1. $\tau$ denotes the EXE state update interval.

\begin{tabular}{lllllllll}
\hline$\tau[\mathrm{ps}]$ & $\begin{array}{l}\beta \text {-sheet } \\
\text { Pop. }\end{array}$ & Error & Pop. & Error & Pop. & Error & Pop. & Error \\
\hline 0.2 & 0.271 & 0.008 & 0.34 & 0.01 & 0.149 & 0.005 & 0.023 & 0.004 \\
0.4 & 0.272 & 0.006 & 0.34 & 0.01 & 0.147 & 0.006 & 0.021 & 0.003 \\
0.6 & 0.270 & 0.009 & 0.330 & 0.006 & 0.150 & 0.004 & 0.020 & 0.003 \\
1.0 & 0.28 & 0.01 & 0.350 & 0.009 & 0.138 & 0.009 & 0.0216 & 0.0005 \\
2.0 & 0.281 & 0.005 & 0.35 & 0.01 & 0.139 & 0.005 & 0.021 & 0.005 \\
5.0 & 0.278 & 0.008 & 0.355 & 0.007 & 0.137 & 0.005 & 0.026 & 0.007 \\
\hline
\end{tabular}


a)

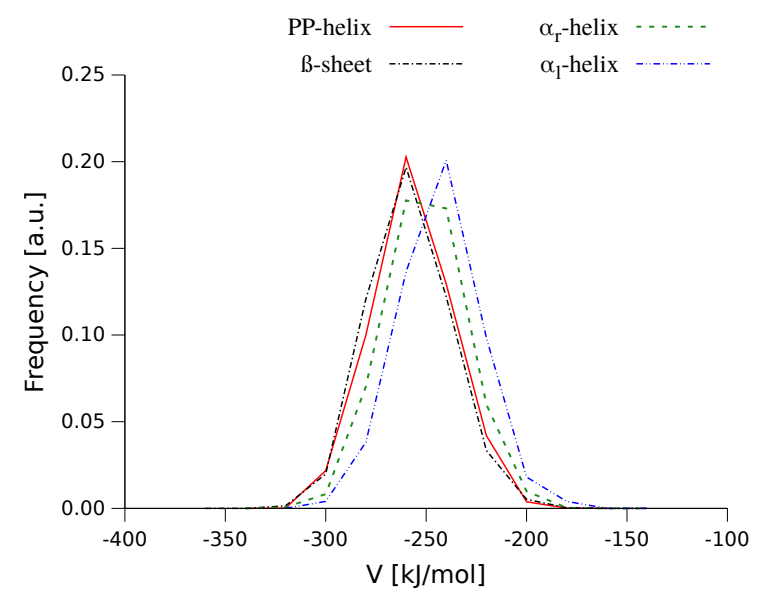

b)

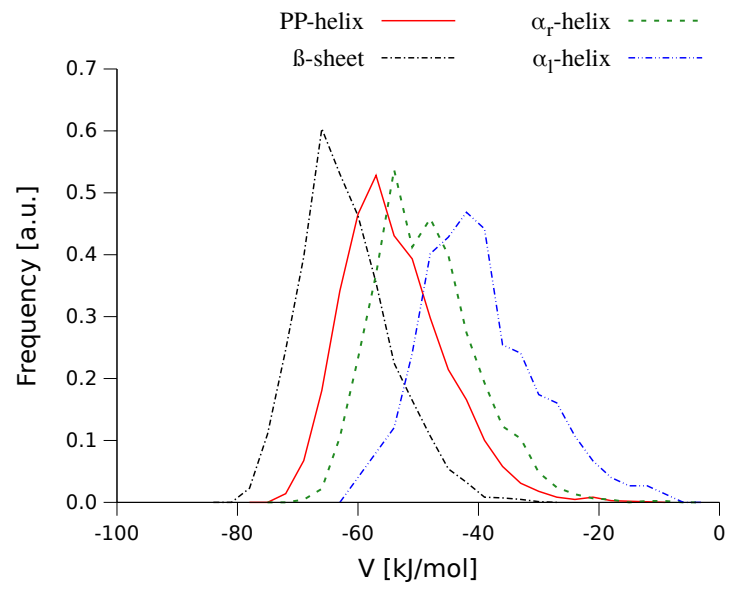

Figure S2: Potential energy distributions for the four secondary structure elements at an effective temperature of $300 \mathrm{~K}$ : a) intra-peptide potential plus peptide-water interactions (short-range Coulomb and short-range van der Waals), b) intra-peptide potential only. Data taken from an EXE run with the following settings: integrator = velocity Verlet, thermostat $=$ Nosé-Hoover chain, $\tau=2$ ps.

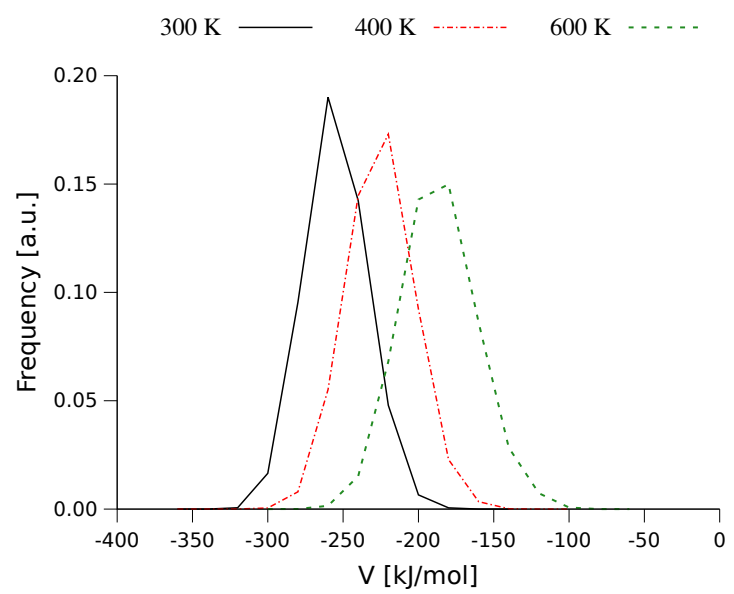

Figure S3: Potential energy distributions (intra-peptide potential plus peptide-water interactions) sampled at the three effective temperatures (states). To obtain comparable data, the potential energies of all configurations in all states were calculated using the unmodified potential energy function corresponding to $300 \mathrm{~K}$. Data taken from an EXE run with the following settings: integrator $=$ velocity Verlet, thermostat $=$ Nosé-Hoover chain, $\tau=2$ ps. 


\section{References}

(1) Abraham, M. J.; Murtola, T.; Schulz, R.; Páll, S.; Smith, J. C.; Hess, B.; Lindahl, E. GROMACS: High performance molecular simulations through multi-level parallelism from laptops to supercomputers. SoftwareX 2015, 1-2, 19-25.

(2) Páll, S.; Abraham, M. J.; Kutzner, C.; Hess, B.; Lindahl, E. In Solving Software Challenges for Exascale; Markidis, S., Laure, E., Eds.; 2015; Vol. 8759; pp 3-27.

(3) Pronk, S.; Páll, S.; Schulz, R.; Larsson, P.; Bjelkmar, P.; Apostolov, R.; Shirts, M. R.; Smith, J. C.; Kasson, P. M.; van der Spoel, D.; Hess, B.; Lindahl, E. GROMACS 4.5: a high-throughput and highly parallel open source molecular simulation toolkit. Bioinformatics 2013, 29, 845-854.

(4) Hess, B.; Kutzner, C.; van der Spoel, D.; Lindahl, E. GROMACS 4: Algorithms for Highly Efficient, Load-Balanced, and Scalable Molecular Simulation. J. Chem. Theory Comput. 2008, 4, 435-447.

(5) van der Spoel, D.; Lindahl, E.; Hess, B.; Groenhof, G.; Mark, A. E.; Berendsen, H. J. C. GROMACS: Fast, Flexible, and Free. J. Comput. Chem. 2005, 26, 1701-1718.

(6) Lindahl, E.; Hess, B.; van der Spoel, D. GROMACS 3.0: a package for molecular simulation and trajectory analysis. J. Mol. Model 2001, 7, 306-317.

(7) Berendsen, H. J. C.; van der Spoel, D.; van Drunen, R. GROMACS: A message-passing parallel molecular dynamics implementation. Comp. Phys. Comm. 1995, 91, 43-56.

(8) Cornell, W. D.; Cieplak, P.; Bayly, C. I.; Gould, I. R.; Merz, K. M.; Jr.,; Ferguson, D. M.; Spellmeyer, D. C.; Fox, T.; Caldwell, J. W.; Kollman, P. A. A Second Generation Force Field for the Simulation of Proteins, Nucleic Acids, and Organic Molecules. J. Am. Chem. Soc. 1995, 117, 5179-5197. 
(9) Hornak, V.; Abel, R.; Okur, A.; Strockbine, B.; Roitberg, A.; Simmerling, C. Comparison of Multiple Amber Force Fields and Development of Improved Protein Backbone Parameters. Proteins: Struct., Funct., Bioinf. 2006, 65, 712-725.

(10) Best, R. B.; Hummer, G. Optimized Molecular Dynamics Force Fields Applied to the Helix-Coil Transition of Polypeptides. J. Phys. Chem. B 2009, 113, 9004-9015.

(11) Abascal, J. L. F.; Vega, C. A general purpose model for the condensed phases of water: TIP4P/2005. J. Chem. Phys. 2005, 123, 234505.

(12) Terakawa, T.; Kameda, T.; Takada, S. On Easy Implementation of a Variant of the Replica Exchange with Solute Tempering in GROMACS. J. Comput. Chem. 2011, 32, $1228-1234$.

(13) Hess, B.; Bekker, H.; Berendsen, H. J. C.; Fraaije, J. G. E. M. LINCS: A Linear Constraint Solver for Molecular Simulations. J. Comput. Chem. 1997, 18, 1463-1472.

(14) Miyamoto, S.; Kollman, P. A. SETTLE: An Analytical Version of the SHAKE and RATTLE Algorithm for Rigid Water Models. J. Comput. Chem. 1992, 13, 952-962.

(15) Páll, S.; Hess, B. A flexible algorithm for calculating pair interactions on SIMD architectures. Comp Phys Comm 2013, 184, 2641-2650.

(16) Essmann, U.; Perera, L.; Berkowitz, M. L.; Darden, T.; Lee, H.; Pedersen, L. G. A smooth particle mesh Ewald method. J. Chem. Phys. 1995, 103, 8577-8593.

(17) Bussi, G.; Donadio, D.; Parrinello, M. Canonical sampling through velocity rescaling. J. Chem. Phys. 2007, 126, 014101.

(18) Berendsen, H. J. C.; Postma, J. P. M.; van Gunsteren, W. F.; DiNola, A.; Haak, J. R. Molecular dynamics with coupling to an external bath. J. Chem. Phys. 1984, 81, 36843690. 
(19) Nosé, S. A molecular dynamics method for simulations in the canonical ensemble. Mol. Phys. 1984, 52, 255-268.

(20) Hoover, W. G. Canonical dynamics: Equilibrium phase-space distributions. Phys. Rev. A 1985, 31, 1695-1697.

(21) Liu, J. S. Peskun's theorem and a modified discrete-state Gibbs sampler. Biometrika 1996, 83, 681-682.

(22) Wang, F.; Landau, D. P. Efficient, Multiple-Range Random Walk Algorithm to Calculate the Density of States. Phys. Rev. Lett. 2001, 86, 2050-2053.

(23) Andersen, H. C. Molecular dynamics simulations at constant pressure and/or temperature. J. Chem. Phys. 1980, 72, 2384-2393. 